\title{
Experience of Pregnant Mothers on Preconception Care Among Antenatal Care Attendants in Sendafa Town Public Health Facilities, Ethiopia, 2020
}

\author{
Ketema Gurmu ${ }^{1,}$, , Wassie Negash ${ }^{2}$, Zalalem Kaba ${ }^{3}$, Robert Wondimu ${ }^{1}$ \\ ${ }^{1}$ Sendafa Beke Town Health Office, Sendafa Beke, Ethiopia \\ ${ }^{2}$ Department of Public Health, Institute of Health Sciences, Debre Berhan University, Debre Berhan, Ethiopia \\ ${ }^{3}$ East Wollega Zonal Health Office, Nekemte, Ethiopia \\ Email address: \\ ketemagurmu2021@gmail.com (K. Gurmu),Wassienegashh@gmail.com (W. Negash), kabazalalem@gmail.com (Z. Kaba), \\ robertwondimu@gmail.com (R. Wondimu) \\ ${ }^{*}$ Corresponding author
}

\section{To cite this article:}

Ketema Gurmu, Wassie Negash, Zalalem Kaba, Robert Wondimu. Experience of Pregnant Mothers on Preconception Care Among Antenatal Care Attendants in Sendafa Town Public Health Facilities, Ethiopia, 2020. World Journal of Public Health. Vol. 6, No. 3, 2021 , pp. 99-109. doi: $10.11648 /$ j.wjph.20210603.14

Received: June 23, 2021; Accepted: July 21, 2021; Published: Published: August 9, 2021

\begin{abstract}
Preconception care is the provision of biomedical, behavioral, and social health interventions to women and couples before conception occurs. Today, there is already a global consensus to incorporate preconception care as one of the continuum of reproductive health services which could decrease $71 \%$ of unwanted pregnancies, thereby eliminating 22 million unplanned births, 25 million induced abortions, and 7 million miscarriages. It was assessed the experience of preconception care and associated factors among pregnant Women attending Antenatal care at public health facilities in Sendafa town, Ethiopia from January 01-February 30, 2020. A cross-sectional study design was employed among 617 pregnant women attending antenatal care in public healthcare facilities. A systematic random sampling technique was employed. Interviewer administered structured questionnaire was used. The data were analyzed by using SPSS version 20 and $\mathrm{P}<0.05$ at $95 \%$ confidence interval was taken as a statistically significant association. The majority $(525,88.7 \%)$ of the women had poor knowledge and only $67(11.3 \%)$ had good knowledge of preconception care services. Pregnant women who had partner support on preconception care service, previous history of abortion, and women diagnosed with anemia were significantly associated with the experience of preconception care services. An experience of pregnant mothers on preconception care services was low and the study found that the knowledge level of study participants on preconception care services was very poor. Health education should be given for all women in the reproductive age group on preconception care services and male involvement should be encouraged for the provision of partner support.
\end{abstract}

Keywords: Preconception Care, Sendafa Town, Pregnant Mothers, Ethiopia

\section{Introduction}

Preconception care (PCC) is the provision of the biomedical, behavioral and social health interventions to women and couples before conception occurs [1]. PCC is particularly important as fetal development commences in the first trimester of pregnancy, before many women are aware of their pregnancy [2]. This care recognizes that many adolescent girls and young women will be thrust into motherhood without the knowledge, skills or support they need; and that by promoting health and providing preventive care, for better outcomes for them and their children [3]. Evidence strongly suggests that earlier care before pregnancy leads to improved women's health and improved pregnancy outcome for both the mother and the newborn [4].

PCC can reduce death among mothers and children, prevent unplanned and unwanted pregnancies, prevent delivery complications, prevent stillbirth, prevent Low birth weight and preterm labor, prevent congenital anomalies and neonatal infections, prevent stunting, and prevent mother to 
child transmission of human immunodeficiency virus or sexually transmitted infections (HIV/STIs) [5]. Every year, in the past 25 years, about half a million women die due to maternal causes and the sum of the consecutive maternal death reported from the year 1990-2015 results in 10.9 million estimated maternal deaths [6]. The risk of maternal and infant mortality and pregnancy-related complications can be reduced by increasing access to quality preconception (before pregnancy) and inter conception (between pregnancies) care [7].

The lately identified but effective evidence-based intervention among the continuum of the spectrum of the maternal and child health services is PCC which targeting the critical periods of the infant's organ formation [8]. Since a majority of women are unaware of the effects of their own health conditions and health-related behaviors may have effects on the fetus, they entering in a pregnancy with various preconception risk factors to adverse pregnancy outcomes (APO) [9]. Different articles show that the women's PCC knowledge and practice in developing countries including Africa is low [10-12]. In Ethiopia there are no formal preconception clinics established in health care settings. Yet, PCC is introduced during maternal and child health services like-consultations for contraception, follow-up for chronic diseases, follow up for immunizations services and during post natal care (PNC) services.

Maternal mortality is still high in the Sub-Saharan Africa where Ethiopia falls, despite the adoption of the sustainable development goal (SDG) number three whose objective is to reduce the global maternal mortality ratio from 216 per 100,000 live births to less than 70 per 100,000 live births by the year 2030 . More than $90 \%$ or more of maternal and child mortality remains concentrated in South Asia and SubSaharan Africa, countries that simply lack to deliver existing, effective interventions that could prevent many of these deaths [13]. The Millennium development goal (MDG) report 2014 indicates that the proportion of mothers who do not survive childbirth compared to those who survive childbirth in the developing regions is 14 times higher than in developed nations [14]. The magnitudes of the globally reported APOs related to the fetus and the child are still high, every year 4 million neonatal deaths and 2.7 million stillbirths are reported, of the 4 million annually reported neonatal death, three-fourths $(75 \%)$ are happening during the first weeks of the newborns life [15]. Nearly all of APOs are reported from low-income countries which account the vast majority (99\%) of all the maternal death, more than $60 \%$ of preterm birth, $96.5 \%$ of low birth weight (LBW) and $99 \%$ of neonatal death are happening in developing countries especially in the Sub-Saharan Africa including Ethiopia [16].

According to the Ethiopian demographic health survey (EDHS) 2016 report, 22\% of women age 15-49 years are thin with body mass index (BMI) less than 18.5 , while $8 \%$ are overweight or obese and the burdens of APO in the country and the indicators are still unacceptably high that $13 \%$ of infants weighed less than 2.5 kilogram at birth and the mortality rate of neonates, infants, and under five-children has been 29, 48, and 67 per 1000 live births respectively [17].

In Ethiopia, the existing APO preventive strategies are highly reliant on delivery of antenatal care (ANC), PNC, and child health services. But, with these interventions the health care providers are acting too late after the conception happened and after the women aware that she is pregnant. The first ANC visit unfortunately happens after the 12 weeks of gestation. The early gestational weeks are the missed periods which are critical to the development of the fetal organs. The irreversible and major damages happening to the fetus are happening during this critical period and that's why the existing interventions are too late to solve APOs happening during the critical periods [7, 18]. These will be the obstacle for Ethiopia to achieving Health Sector Transformation Plan two and SDG targets (that is reducing maternal and neonatal mortality below 199 in 2019/20 and 77 in 2030 per $10^{5}$ live births and neonatal mortality less than 10 per 1000 live births) $[19,20]$. Thus moving towards PCC are the potential for earlier risk assessment and intervention that can benefit the woman or couple even before pregnancy and ensure the healthiest possible start for the newborn child [8].

Studies showed that only less than one-third of women of childbearing age visited the health institutions and speak with a health care provider prior to pregnancy about their health status and its potential impact on pregnancy outcome [21]. The studies conducted in Sri Lanka, Nigeria, Sudan and Zambia showed that the women's experience on PCC is very low [22-25]. In one study, it was reported that less than $50 \%$ of women supplement their diet with folate during the preconceptions period, even though, there is strong evidence that folate supplementation reduces the risk of neural tube defects (NTDs) by $80 \%$ and only $53 \%$ of women who had heard from health care providers [26].

Reproductive life planning through PCC could decrease $71 \%$ of the unwanted pregnancy, thereby eliminating 22 million unplanned births, 25 million induced abortion and 7 million miscarriages $[27,28]$. Therefore, in settings where there is low awareness of PCC, promotion of PCC among reproductive age group women is important to boost maternal health care services and to reduce complications during ANC, institutional delivery and PNC. In Ethiopia, little is known about women's experiences on PCC and how women have been preparing for pregnancy. Despite our rigorous literature review and experience, there is limited evidence that tells about the Ethiopian women's experience on PCC. The published articles that assessed women's PCC knowledge in Northern and Southern Ethiopia reported poor PCC knowledge [29, 30]. However, another article that assessed women's experiences on PCC utilization in Debre Berhan town (13.4\%), Debre Markos (8.4\%), west Shoa Oromia (38.2\%) and Mekelle town $(18.2 \%)$, the studies concluded that, women's experience on PCC utilization was very low [31-34].

In previous studies factors influenced women experience on PCC were socio demographic factors, unavailability of PCC unit, family support and obstetric factors. However, another factors associated with women's experience on PCCknowledge related factors on it and history of chronic 
diseases have not been well understood, and there is no research conducted on women's experience on PCC services in our study area. Thus, this study aimed to assess experience of pregnant women on PCC services and associated factors among pregnant mothers attending ANC in public health facilities in Sendafa town, Ethiopia

\section{Methods and Materials}

\subsection{Study Area and Period}

This study was conducted in Sendafa town public healthcare facilities. Sendafa town is found in Oromia regional state administration and situated 39 kilometers north East of Addis Ababa (the nation capital). The town is currently divided into three administrative kebeles (the smallest administrative unit in Ethiopia). According to the 2019, Sendafa town administration estimation report; it has 56,652 populations (male-27,759, and female-28,893). Among this about 12,463 are reproductive age group (15-49 years) women. The town has one senior secondary school; one public primary hospital, two public health center, one health post, and twelve private medium clinics. During the study period, there were 300 health professionals working in the town health facilities. According to Sendafa town health office report in the last fiscal year before the study the percentage of at least one ANC visit was $100 \%$, and at least four ANC visit was $90 \%$. The proportion of pregnant women tested for syphilis was $100 \%$, contraceptive acceptance rate (CAR) was $85 \%$, and proportion of infant who received all vaccines was $91 \%$, proportion of infant whose mothers had protective doses of TT against neonatal tetanus (NNT) was $83 \%$ and number of still birth in last fiscal year before the study was 8 (eight). The study was conducted from January 01-February 30, 2020.

\subsection{Study Design}

An institutional based cross-sectional study design was employed.

\subsection{Population}

\subsubsection{Source Population}

All pregnant women (PW) who were attending ANC in public health facilities in Sendafa town were taken as source population.

\subsubsection{Study Population}

All PW attending ANC during study period and fulfill the inclusion criteria in public health facilities in Sendafa town.

\subsection{Eligibility Criteria}

\subsubsection{Inclusion Criteria}

All PW who were attending ANC during study period in public health facilities and living in Sendafa town for more than six month.

\subsubsection{Exclusion Criteria}

PW who have hearing problem, unable to talk and not living in Sendafa town more than six month were excluded.

\subsection{Sample}

\subsubsection{Sample Size Determination}

The designed Sample size was estimated using a single population proportion formula for the first objective and by using the software Epi info version 7 for the second objective. For the first objective the sample size calculated with following assumption:-95\% confidence interval (CI) and anticipated prevalence of women's experience on PCC is $38.2 \%$ [34], 4\% margin of error. Then, the calculated sample size was:

$$
\mathrm{n}=\frac{(Z \delta / 2)^{2} * P(1-P)}{(d)^{2}}
$$

Where; $\mathrm{n}=$ Sample size, $\mathrm{Z}=$ the standard normal deviation at $95 \% \mathrm{CI}=1.96$

$\mathrm{P}=$ anticipated proportion of $\mathrm{PCC}$ experience among ANC attendants; $38.2 \%(0.382), \mathrm{d}=$ margin of error that can be tolerated, $4 \%(0.04)$. Then the calculated sample size of:

$$
\mathrm{n}=\frac{(1.96)^{2} * 0.382(1-0.382)}{(0.04)^{2}}=561
$$

So, the required sample size was:-561; and adjusted nonrespondents rate of $10 \%$, the final sample was: $561 * 10 \%=56$ and then; $561+56=617$. The sample size for the second objective was calculated by using Epi info version7 software by taking $80 \%$ power and $95 \% \mathrm{CI}$. Therefore, the calculated sample size for the second objective is lower than the first objective and then the final sample size for this study was 617 .

\subsubsection{Sampling Technique and Procedure}

All public health facilities in Sendafa town administration was included in the study. A sample size of 617 was proportionally allocated to three public health facilities (Sendafa primary hospital, Sendafa town health center and Walgawo health center) based on the two last month ANC attendant report before the study. Within each facility PW was selected by systematic random sampling technique. The total two month expected number of ANC attendants in Sendafa town administration was 1,260 (420, 486, and 354 in Sendfa primary hospital, Sendafa town health center, and walgawo health center respectively). The sampling interval was determined to select the participant. The last month an ANC attendant in Sendafa town was 630 and then the sampling interval was $2(1,260 / 630, K=2)$. The study participants from each health facilities was selected based on this interval [Figure 1]. 


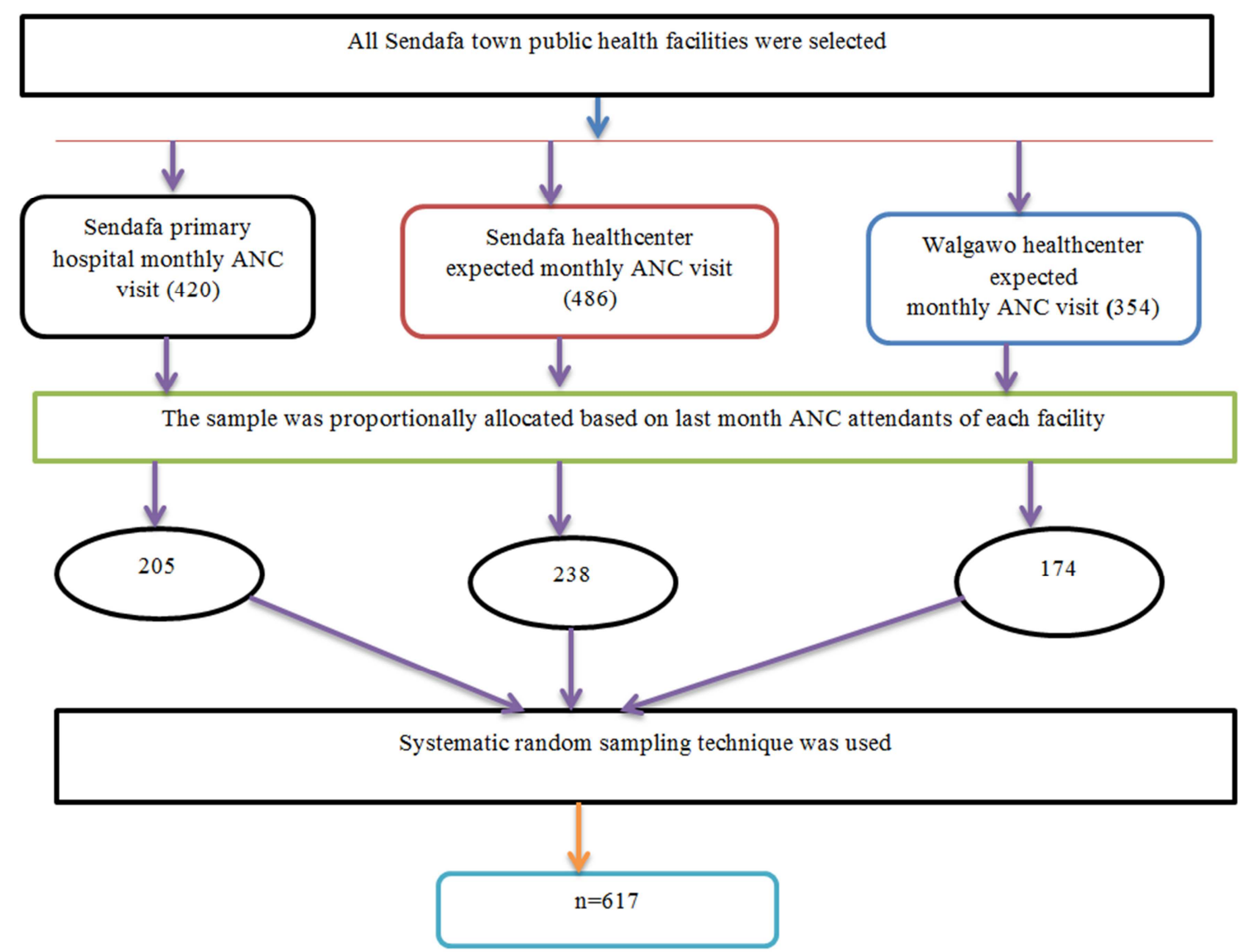

Figure 1. Schematic presentation of sampling technique of experience of PW on PCC in public health facilities, Sendafa town, Ethiopia, 2020.

\subsection{Data Collection Tool and Method}

\subsubsection{Data Collection Tool}

The tool were adapted from previous literatures, Center for effective practice of preconception health care tool (CEP) and then modified according to local context based on objectives of the study [29, 31, 32, 34-36]. Interviewer administered questionnaire were used to collect quantitative data from the respondents. The questionnaire first prepared in English language and then translated to local Afaan Oromo (regional language) before data collection. The study questionnaire were consists of five parts. Part one:-socio demographic information, part two:-knowledge based information, the third part contains questions on experience of PCC, the fourth parts were about obstetric history, and the last part was about history of chronic diseases in each subsection. The guide were adapted from previous literatures and modified based on the objective of the study [37]. The inquiry mainly contains women's experience on PCC, knowledge on PCC and factors influence experience of women on PCC services.

\subsubsection{Data Collection Method}

An interviewer administrated structured questionnaire was employed to collect the data. Three Bachelor of Science (BSc) Midwives as data collectors and one public health officer as supervisor were trained on data collection procedures. First, the questionnaire was prepared in English language and then translated into Afaan Oromo (regional language) and then transcribed back to English properly by expert to check its consistency.

\subsection{Study Variables}

\subsubsection{Dependent Variables}

Experiences on preconception care.

\subsubsection{Independent Variables}

a) Socio-demographic factors [Age, Educational status and husband's educational status Occupation and Marital status].

b) Obstetric factors [gravidity, parity, family planning use, partner support and bad obstetric history].

c) Knowledge on PCC [knowledge on benefits of PCC, knowledge on PCC components].

d) History of maternal chronic disease [diabetic mellitus hypertension, asthma, Epilepsy, syphilis, anemia and HIV/AIDS].

\subsection{Operational Definition}

a) Preconception care experience:- If women received at least one types of interventions either advice or treatment and life style modification, care women received regarding components of PCC (screened for any disease and get treatment, take folic acid, take vaccine, get counseling, cessation of alcohol and cigarette smoking, 
planned pregnancy) before being pregnant [38].

b) Knowledge on PCC:-Women's level of knowledge on PCC was measured based on correct response using seven PCC knowledge based questions. Each correct and incorrect response was scored one and zero point respectively [29].

c) Preconception care components: - In this study PCC components are HIV testing and counseling, STI screening and treatment, chronic medical condition screening and treatment, nutrition counseling and ferrous supplementation, immunization (TT), Advice on cessation of alcohol and advice on cessation of cigarette smoking, and family planning [32].

\subsection{Data Processing}

\subsubsection{Data Quality Control}

Training was given for data collectors and supervisor by principal investigator on how to fill the questionnaire and method of approaching study participants for one day. The filled questionnaires was Checked for completeness by the supervisors at the site of data collection and finally by principal investigator. The Questionnaires was pre-tested on $5 \%$ of study participants in LegeTafo health center two weeks before the actual data collection period to check the consistency of instruments, adequacy of variable and orders of the question. After pre-test, amendment was made accordingly. Reliability and validity testing of the instrument was undertaken to check the internal consistency. Reliability test was performed for each PCC experience based questions using Cronbach's alpha test.

\subsubsection{Measurement}

The knowledge level of the study subjects was determined using dichotomous scale. A total of seven knowledge statements was ranging from whether one had heard about PCC services, mentioned the components of PCC services and preconception health and behavior risk factors which affect the fetus. Each Yes answer statement was earn the participants 1 (one) point and No answer was earn 0 (zero) point. The calculated single knowledge factor was categorized into two ordinal categories. Respondents who score less than four knowledge based questions was categorized as poor knowledge on PCC services whereas participants who was scored greater or equal to four knowledge based questions was categorized as good knowledge on PCC services [30, 39]. There were 11 (eleven) PCC service experience based questions. Overall experience of PCC services of the study participants was assessed; if the women received at least one types of interventions either advice or treatment and life style modification; care women received regarding components of PCC (screened for any disease and get treatment, take folic acid, take vaccine, get counseling, cessation of alcohol and cigarette smoking, planned pregnancy) before being pregnant.

\subsubsection{Data Analysis Method}

The data was coded and entered into Epi-data version 4.2.0 and exported to SPSS version 20.0 for analysis. Descriptive statistics like mean, frequency and proportion was used and findings was presented in texts, tables and charts. Logistic regression including bivariate and multivariate analysis was used to examine association between dependent and independent variables. Bivariate analysis was performed using binary logistic regression at $\mathrm{P}<0.25$ was criteria to select candidate variables for multivariable analysis. Multivariable logistic regression analysis was done to adjust for possible confounding variables at $\mathrm{P}<0.05$, AOR at $95 \%$ CI was used in judging the statistical significance of that association.

\subsection{Ethical Consideration}

Ethical clearance was obtained from Debre Berhan University College of health science department of Public health Ethical review Committee. Permission to gather data was obtained from Sendafa town Health Bureau, Sendafa town public administration and from each health center administrative office. An information sheet was provided for each study participant on the introductory part of the questionnaires and interview topic guide that further explains the study purpose and confidentiality of the research information. In addition, oral consent was sought from each study participant prior to data collection.

\section{Result}

\subsection{Socio-demographic Characteristics of the Study Participants}

A total $617 \mathrm{PW}$ on $\mathrm{ANC}$ visits were expected to participants on the study and 592 were responded to the study (95.8\% response rate). The mean age of the study participants was 29.08 ( \pm SD 5.72). Majority of the respondents were married 443 (74.8\%). Almost about one-third (185, 31.2\%) of the respondents were completed primary school (1-8 grade). Among the total study participants $240(40.5 \%)$ were house wives in occupation, and one-fifth $(126,21.3 \%)$ of them were government employers [Table 1].

\subsection{Knowledge of Pregnant Womens on Preconception Care}

Almost more than one-third (206, 34.8\%) of participants heard about PCC and the most source of information about PCC was health facilities $(121,20.4 \%)$ followed by information from community $56(9.5 \%), 23(3.9 \%)$ from social media, and $8(1.4 \%)$ from religious institutions. Most of the study participants did not know the components of PCC $(495,83.6 \%)$ and only $97(16.4 \%)$ of the participants know the components of PCC service. Among the respondents $164(27.7 \%)$ know the TT vaccination before conception and 118 (19.9\%) know about chronic medical condition screening during PCC period. Most of the respondents $506(85.5 \%)$ did not know about the effects of alcohol and cigarettes smoking on pregnancy before conception. Five hundred thirty one $(531,89.7 \%)$ did not 
know weight monitoring and folic acid use during preconception period. Almost around one-third (190, 32.1\%) of the respondents know about STI screening before conception [Table 2].

Women's knowledge on PCC were measured based on correct response using seven knowledge questions. The calculated single knowledge factor was taken, and then categorized into two categories. Respondents who scored less than the mean were categorized as poor knowledge whereas who were scored greater or equal to the mean was categorized as good PCC knowledge. The majority of the study participants $(525,88.7 \%)$ of the women had poor knowledge and only $(67,11.3 \%)$ had good knowledge on PCC.

Table 1. Socio demographic characteristics of women's experience on PCC among PW in Sendafa town public health facilities, Ethiopia, 2020 [N=592].

\begin{tabular}{llll}
\hline Variables & Response category & Frequency (N) & Percent (\%) \\
\hline \multirow{4}{*}{ Age (in years) } & $15-24$ & 122 & 21 \\
& $25-34$ & 328 & 55 \\
& $35-44$ & 142 & 24 \\
& $\geq 45$ & 0 & 0 \\
Marital status & Married & 443 & 75 \\
& Divorced & 69 & 6 \\
& Single & 41 & 4 \\
Educational Status & Separated & 23 & 1 \\
& No formal education & 16 & 29 \\
& Primary school & 169 & 31 \\
Religion & Secondary school (2ndry) & College and above & 20 \\
& Orthodox & 185 & 20 \\
& Muslim & 120 & 49 \\
& Protestant & 291 & 28 \\
Occupation & Wakefana & 162 & 21 \\
& House wife & 129 & 2 \\
& Private Business & 9 & 41 \\
& Government employee & 240 & 27 \\
\end{tabular}

Table 2. Pregnant Womens knowledge on PCC components and importance among ANC attendants in Sendafa town public health facilities, Ethiopia, 2020 [N=592].

\begin{tabular}{|c|c|c|c|}
\hline Knowledge Variables & Response & Frequency $(\mathbf{N})$ & Percent (\%) \\
\hline \multirow{2}{*}{ Ever heard about PCC } & Yes & 206 & 35 \\
\hline & No & 386 & 65 \\
\hline \multirow{2}{*}{ Know that a women should be on FP before conception } & Yes & 253 & 43 \\
\hline & No & 339 & 57 \\
\hline \multirow{2}{*}{ Know that a women should vaccinated against Tetanus before conception } & Yes & 164 & 28 \\
\hline & No & 428 & 72 \\
\hline \multirow{2}{*}{ Know that women should be screened for chronic medical illness } & Yes & 118 & 20 \\
\hline & No & 474 & 80 \\
\hline \multirow{2}{*}{ Know that a women should stop alcohol drinking and cigarette smoking before conception } & Yes & 86 & 15 \\
\hline & No & 506 & 85 \\
\hline \multirow{2}{*}{ Know that a women should undergo weight monitoring } & Yes & 61 & 10 \\
\hline & No & 531 & 90 \\
\hline \multirow{2}{*}{ Know that use folic acid before conception } & Yes & 61 & 10 \\
\hline & No & 531 & 90 \\
\hline \multirow{2}{*}{ Know that a women should screened for STI/HIV } & Yes & 190 & 32 \\
\hline & No & 402 & 68 \\
\hline \multirow{2}{*}{ Know the components of PCC and its importance } & Yes & 97 & 16 \\
\hline & No & 495 & 84 \\
\hline
\end{tabular}

\subsection{Source of Information on Preconception Care Services}

Majority of the study participants (495, 83.6\%) never knew services given in the period of PCC. Health facilities were the major source of information $(58 \%)$ followed by community $(27 \%)$, mass media $(11 \%)$, and the least source of information was religious institutions $(4 \%)$.

\subsection{Obstetric Characteristics of Study Participants}

One hundred eight (18.2\%) Womens were primigravida, and $484(81.8 \%)$ of them were multigravida whereas $218(36.8 \%)$ of the participants were primipara and $255(43.1 \%)$ of them were multipara. In three hundred seventy one $(62.7 \%)$ of the mother's recent pregnancy were planned. About 216 (36.5\%) participants were attended early ANC (that is before three months of 
gestation). Among the study participants $(164,27.7 \%)$ were experienced obstetric problems. Three hundred ninety (53.9\%) of the mothers were reported that their partners were supported them for PCC services [Table 3].

Table 3. Obstetric characteristics of study participants among ANC attendants in Sendafa town public health facilities, Ethiopia, 2020 [N=592].

\begin{tabular}{|c|c|c|c|}
\hline Obstetric Characteristics & Response category & Frequency (N) & Percent (\%) \\
\hline \multirow{3}{*}{ Parity } & Primipara & 218 & 37 \\
\hline & Multipara & 255 & 43 \\
\hline & Nulipara & 119 & 20 \\
\hline \multirow{2}{*}{ Gravidity } & Primigravida & 108 & 18 \\
\hline & Multigravida & 484 & 82 \\
\hline \multirow{3}{*}{ Previous obstetric problem } & Abortion & 77 & 13 \\
\hline & Congenital malformation & 25 & 4 \\
\hline & Neonatal death & 19 & 3 \\
\hline \multirow{2}{*}{ Planned pregnancy } & Yes & 371 & 63 \\
\hline & No & 221 & 37 \\
\hline \multirow{2}{*}{ Mothers attended Early ANC } & Yes & 216 & 37 \\
\hline & No & 376 & 63 \\
\hline \multirow{2}{*}{ Partners support for PCC } & Yes & 319 & 54 \\
\hline & No & 273 & 46 \\
\hline
\end{tabular}

\subsection{Experience of Pregnant Women on Preconception Care}

About $(345,58.3 \%)$ of pregnant Women have experience of PCC and 247 (41.7\%) have no experience on it. Among the study participants only $(319,53.9 \%)$ had support from their husbands for preconception screening. Almost more than half $(371,62.7 \%)$ of the pregnant women current pregnancy were planned. Most of the study participants 484
$(81.1 \%)$ used at least one components PCC services and the most known components of PCC was family planning (266, $44.9 \%)$, TT vaccinated before pregnancy $(120,20.3 \%)$, and about $(58,9.8 \%)$ screened for chronic medical condition. Only $(44,7.4 \%)$ pregnant women were monitor their weight before pregnancy and $243(41 \%)$ of them were screened for HIV/AIDS before conception [Table 4].

Table 4. Experience of Pregnant Women on preconception care services among ANC attendants in Sendafa town public health facilities, Ethiopia, 2020 $[N=592]$.

\begin{tabular}{|c|c|c|c|}
\hline PCC experience variables & Response category & Frequency (N) & Percent (\%) \\
\hline \multirow{2}{*}{ FP use before conception } & Yes & 266 & 45 \\
\hline & No & 326 & 55 \\
\hline \multirow{2}{*}{ Immunized against tetanus before conception } & Yes & 120 & 20 \\
\hline & No & 472 & 80 \\
\hline \multirow{2}{*}{ Screened for any medical condition during preconception } & Yes & 58 & 10 \\
\hline & No & 534 & 90 \\
\hline Advised on the effects of alcohol and cigarette smoking on pregnancy before conception & Yes & 109 & 18 \\
\hline \multirow{2}{*}{ Weight monitored before conception } & Yes & 44 & 7 \\
\hline & No & 548 & 93 \\
\hline \multirow{2}{*}{ Screened for HIV/AIDS before current pregnancy } & Yes & 243 & 41 \\
\hline & No & 349 & 59 \\
\hline \multirow{2}{*}{ Early attended ANC at first three month of gestation } & Yes & 216 & 37 \\
\hline & No & 376 & 63 \\
\hline Screened for another STI and Infectious disease & Yes & 119 & 20 \\
\hline Consumed folic acid supplementation three months before conception & No & 540 & 91 \\
\hline
\end{tabular}

\subsection{Factors Associated with Experience of Pregnant Womens on Preconception Care Services}

In binary logistic regression, 13 (thirteen variables) were significantly associated with dependent variable. These were:-Women's knowledge on preconception care, family size, previous history of abortion, being diagnosed for anemia, being diagnosed for diabetic mellitus, being diagnosed for hypertension, traditional and cultural influence, unavailability of the service in health facilities, partner support on PCC, planned pregnancy, ever heard about PCC, husband educational status, and educational status of the respondent were variables identified as candidate for multivariate logistic regression analysis [Table 5].

After controlling for the effect of potential confounding variables using multivariable logistic regression, partner support on PCC services, previous history of abortion and being diagnosed for anemia were remained significantly associated with experience of PCC services with 95\% CI $(\mathrm{P}<0.05)$ [Table 5].

The study reviled that three factors found to be show 
association with women's experience on PCC services. PW who had a partner support on PCC service with 95\%CI, ( $\mathrm{AOR}=3.071-23.397, \mathrm{P}=0.000)$ were 8 (eight times) more likely to have an experience of PCC services than counter parts. This implicate male involvement in PCC service could improve women's service utilization. Women who have previous history of abortion with $95 \% \mathrm{CI},(\mathrm{AOR}=1.55-9.410$, $\mathrm{P}=0.003$ ) were 3 (three times) more likely to have an experience of PCC services than women their counter parts. PW who were diagnosed for Anemia with 95\% CI, ( $\mathrm{AOR}=1.342-13.516, \mathrm{P}=0.014$ ) were 4 (four times) more likely to have an experience of PCC services than women who did not diagnosed for Anemia. Women having previous abortion and diagnosed for anemia may advised for next pregnancy, measure to be taken and more experienced on PCC services [Table 5].

Table 5. Binary logistic regression and multivariable logistic regression analysis of pregnant women Experience on preconception care among ANC attendant in Sendafa town public health facilities, Ethiopia, 2020 [N=592].

\begin{tabular}{|c|c|c|c|c|c|}
\hline \multirow{2}{*}{ Variables } & & \multicolumn{2}{|c|}{ PCC Experience } & \multirow{2}{*}{ COR (95\%) CI } & \multirow{2}{*}{ AOR (95\%) CI } \\
\hline & & Yes & No & & \\
\hline \multirow{2}{*}{ Partner support } & Yes & 227 & 92 & $3.241(2.305,4.556) 0.000 *$ & $8.477(3.071-23.397) 0.000 * *$ \\
\hline & No & 119 & 154 & 1 & $3.826(1.55-9.410) 0.003 * *$ \\
\hline \multirow{2}{*}{ History of abortion } & Yes & 55 & 67 & $1.638(0.871,3.083), 0.029 *$ & \\
\hline & No & 46 & 37 & 1 & \\
\hline \multirow{2}{*}{ Diagnosed for Anemia } & Yes & 52 & 13 & 3.195 (1.699-6.008) 0.022* & $4.259(1.342-13.516) 0.014 * *$ \\
\hline & No & 293 & 234 & 1 & \\
\hline \multirow{2}{*}{ Diagnosed diabetes mellitus } & Yes & 54 & 19 & $0.272(0.158-2.227) 0.004^{*}$ & $1.208(0.422-3.456) 0.725$ \\
\hline & No & 291 & 228 & 1 & \\
\hline \multirow{2}{*}{ Diagnosed for hypertension } & Yes & 62 & 27 & 1.785 (0.244-91.7885) 0.019* & $0.970(0.340-2.772) 0.955$ \\
\hline & No & 283 & 220 & 1 & \\
\hline \multirow{2}{*}{ Knowledge on PCC } & Good & 64 & 3 & $0.054(0.017-0.174) 0.000 *$ & $0.02(0.042,23.23), 0.998$ \\
\hline & Poor & 281 & 244 & 1 & \\
\hline \multirow{2}{*}{ Family size } & $>2$ & 272 & 180 & $1.236(0.0477-1.387) 0.093$ & $1.409(0.902-2.200) 0.132$ \\
\hline & $<2$ & 73 & 67 & 1 & \\
\hline \multirow{2}{*}{ Traditional influence } & Yes & 21 & 3 & $5.272(0.027-5.22) 0.008^{*}$ & $1.657(0.262-10496) 0.592$ \\
\hline & No & 324 & 244 & & \\
\hline \multirow{2}{*}{ Unavailability of service in health facility } & Yes & 110 & 38 & $2.574(0.134-2.574) 0.000 *$ & $0.951(0.301-2.999) 0.931$ \\
\hline & No & 235 & 209 & 1 & \\
\hline \multirow{2}{*}{ Planned pregnancy } & Yes & 237 & 134 & $1.851(0.306-1.851) 0.000 *$ & $0.578(0.248-1.347) 0.204$ \\
\hline & No & 108 & 113 & 1 & \\
\hline \multirow{2}{*}{ Ever heard about PCC } & Yes & 170 & 36 & $(0.037-5.694) 0.000 *$ & $2.115(0.843-5.309) 0.111$ \\
\hline & No & 175 & 211 & 1 & \\
\hline \multirow{2}{*}{ Educational status of mother } & $<2$ ndry & 253 & 219 & $0.716(0.352-2.462) 0.000 *$ & $3.764(0.201-70.411) 0.375$ \\
\hline & $>2$ ndry & 92 & 28 & 1 & \\
\hline \multirow{2}{*}{ Educational status of husband } & $<2$ ndry & 235 & 209 & $0.637(0.388-2.200) 0.000 *$ & $0.154(0.012-1.950) 0.149$ \\
\hline & $>2$ ndry & 110 & 38 & 1 & \\
\hline
\end{tabular}

*Significant at $\mathrm{P}<0.25$, ** Significant at $\mathrm{P}<0.05$, COR (crude odd ratio), AOR (Adjusted odd ratio), P-value for COR, P-value for AOR, 1:-logical reference.

\section{Discussion}

Understanding of PCC is critical for many African countries such as Ethiopia where maternal and prenatal mortality remains high. This facility based cross-sectional study identified factors associated with women experiences on PCC services among PW attending ANC in Sendafa town public health facilities.

The finding of this study showed that an experience of PW on PCC services was 345 (58.3\%). This study found that the overall experience of PCC was higher than the study conducted in Malaysia (45\%), Nepal (26\%), Iraq (40\%), and Debre Markos [11, 40, 41, 32]. This variability may be related to difference in study population, level of education, culture and study setting. However it is lower than the study conducted in Iran (68.8\%), France (85\%), Jordan (85\%) and USA $(76 \%)[12,42,43]$. This discrepancy might be due to the difference in access to information and low relative media coverage, socio economic status and quality of health care system.
This study finding showed that partner support on PCC was associated with experience on PCC services. Women who had a partner support on PCC services are eight times more likely to have an experience of PCC services than who did not have partner support on PCC services $(\mathrm{AOR}=8.477$ (3.071-23.397, $\mathrm{P}=0.000$, CI of 95\%). Study from Egypt, Ethiopia:-Mekelle, and Debre Berhan towns also in line with this study. This may be due to having reproductive health policies including male engagement strategies promoting maternal and child health care services. In addition women who have partner support can discuss more about the importance and benefit of PCC services [31, 32, 44].

The study also indicated that the PW who had previous history of abortion were positively associated with experience of $\mathrm{PCC}$ services with $(\mathrm{AOR}=3.826$ (1.55-9.410) ( $\mathrm{P}=0.003$ ), CI of $95 \%$. Those who have history of abortion were three times more likely to have an experience of PCC services than who did not have history of abortion. This is supported by study conducted in Iraq, Kenya, and Saudi Arabia [41, 37, 45]. This indicating previous APO being associated with increased odds of having PCC services in 
most recent pregnancy. Potentially mothers who had an experience of abortion were more conscious and PCC service compliant in their subsequent pregnancies. This might be due to women who had history of abortion may get pregnancy counseling, including PCC services for next conception.

Being diagnosed for Anemia was also significantly associated significantly associated with women's experience on PCC services (AOR=4.079, CI of 95\% (1,229-13.540) in this study. Women who were being diagnosed for Anemia were four times likely to have an experience of PCC services than who did not diagnose for anemia with $(\mathrm{AOR}=4.259$ (1.342-13.516), 95\% CI, $(\mathrm{P}=0.014)$. But the study from Jordan and China are in contrast with this study showing that no association between being diagnosed for anemia and experience of PCC services [46, 47]. This might be the difference between socio-demographic of the study participants. In this study women who had good knowledge on PCC services were 2 times more likely to experience PCC services than that of poor knowledge. This might be due to an in-depth knowledge of PCC services may increase women's understanding and awareness of their purposes and importance of PCC, thus they use services.

\subsection{Strength of the Study}

In this study participants were recruited from different health facilities, so that the experience of women visiting different health facilities may different reflection variation in PCC services and high response rate. High response rate may reflect the face-to-face recruitment and interview of study participants. Besides, larger sample size was taken and probability sampling technique was employed.

\subsection{Limitation of the Study}

It was purely quantitative and does not have capacity to explore contextual and social factors that may be limiting women in PCC services. It also prone to recall bias as women were asked about pre-pregnancy care for the current pregnancy and this study only relies on the client's experience about their PCC, it would be important to obtain the care providers view and experience on the same issue.

\section{Conclusion and Recommendation}

\subsection{Conclusion}

The finding of this study showed that an experience of pregnant women on PCC services was low and the knowledge level of the study participants on PCC services was very poor. Partner support on PCC services, previous history of abortion and being diagnosed for anemia were factors that associated with experience of PCC services.

\subsection{Recommendation}

Establishing PCC programs which can address all components of PCC services and understand the view of reproductive age group should be existed. Health education for women of reproductive age on PCC services, its components and importance should be given in the health facilities and higher learning institutions by all concerned bodies to promote their knowledge on PCC services and increases its utilization. We should recognize the role Male involvement should be encouraged for provision of partner support. The ministry of Health, regional health bureau, and others concerned bodies should increase public awareness on PCC services through mass media, at health post, churches, and women groups to increase the uptake of PCC services at each level.

Further research should be done on the relationship between partner support and the PCC services. We also recommended that mixed method research, and program evaluation should be undertaken to explore experience of women on PCC services.

\section{Lists of Abbreviation}
a) $A N C \ldots$ Antenatal Care
b) $A P O \ldots$ Adverse Pregnancy Outcome
c) $P W \ldots$...Pregnant Women
d) $P N C$...Postnatal care
e) $P C C \ldots$...Preconception Care

\section{Ethics Approval and Informed Consent}

Ethical clearance was obtained from Debre Berhan University College of health science department of Public health Ethical review Committee. Permission to gather data was obtained from Sendafa town Health Bureau, Sendafa town public administration and from each health center administrative office. An information sheet was provided for each study participant on the introductory part of the questionnaires and interview topic guide that further explains the study purpose and confidentiality of the research information. In addition, oral consent was sought from each study participant prior to data collection.

\section{Consent for Publication}

Not applicable.

\section{Data Availability}

The finding of this study was generated from the data collected and analyzed based on stated methods and materials. The original data supporting this finding are available from the corresponding author on reasonable request.

\section{Competing Interests}

The authors declare that they have no competing interests.

\section{Authors' Contributions}

$K G$ participated in the design of the study, performed the data collection and the statistical analysis and served as the 
corresponding author of the manuscript. $Z K$ and $R W$ supervised the study, ensured quality of the data, assisted in the analysis and interpretation of the data. All authors read and approved the manuscript.

\section{Authors' Information}

1) Ketema Gurmu is Public Health Expert at Ethiopian Police University Health Division, Sendafa Town, Ethiopia.

2) Zalalem Kaba is Water, Sanitation, Hygiene (WASH) and Neglected Tropical Diseases (NTDs) Program Coordinator, Nekemte town, Western Ethiopia.

3) Robert Wondimu is Maternal and Child Health Program Coordinator, Sendafa Town, Ethiopia.

\section{Acknowledgements}

We are grateful to Debre Berhan University for their cooperation during the study. Our deepest gratitude also goes to all data collectors and supervisors without their hard work this work had not been successful. Last but not least our thanks go to all study participants for providing us the valuable information and support.

\section{References}

[1] Mason E, Chandra-Mouli V, Baltag V, Christiansen C, Lassi ZS, Bhutta ZA. Preconception care: Advancing from "important to do and can be done" to "is being done and is making a difference." Reprod Health [Internet]. 2014; 11 (Suppl 3): S8. Available from: http://www.reproductivehealth-journal.com/content/11/S3/S8.

[2] Guideline N. Diabetes in pregnancy: management from preconception to the postnatal period. Published: 25 February 2015. 2019; (February 2015): 55. Available from: https://www.nice.org.uk/guidance/ng3.

[3] Kerber KJ dG-JJ, Bhutta ZA, Okong P, Starrs A LJ. Continuum of care for maternal, newborn, and child health: from slogan to service delivery. Lancet. 370: (9595): 135869.

[4] Dean S, Rudan I, Althabe F, Webb Girard A, Howson C, Langer A, et al. Setting Research Priorities for Preconception Care in Low- and Middle-Income Countries: Aiming to Reduce Maternal and Child Mortality and Morbidity. PLoS Med. 2013; 10 (9).

[5] Braspenningx S, Haagdorens M, Blaumeiser B, Jacquemyn Y, Mortier G. Preconceptional care: a systematic review of the current situation and recommendations for the future. Facts, views Vis ObGyn [Internet]. 2013; 5 (1): 13-25. Available from:

http://www.ncbi.nlm.nih.gov/pubmed/24753925\%0Ahttp://w ww.pubmedcentral.nih.gov/articlerender.fcgi?artid=PMC3987 351.

[6] Alkema L, Chou D, Hogan D, Zhang S, Moller A-B, Gemmill A, et al. National, Regional ang Global levels and trend in MMR between 1990 and 2015. Lancet. 2016; 387 (10017): 462-74.
[7] Kay $\mathrm{J}$ et al. Recommendations to improve preconception healthand health care-United States. A report of the CDC/ATSDR Preconception Care Work Group and the Select Panel on Preconception Care. Atlanta: 2006/55 (RR06): 1-23. $2006 ; 1-23$.

[8] Dean S V, Lassi ZS, Imam AM, Bhutta ZA. Preconception care: Promoting reproductive planning. Reprod Health [Internet]. 2014; 11 (Suppl 3): S2. Available from: http://www.reproductive-health-journal.com/content/11/S3/S2 .

[9] Mastroiacovo P, Nilsen RM, Leoncini E, Gastaldi P, Allegri V, Boiani A, et al. Prevalence of maternal preconception risk factors: an Italian multicenter survey. Ital J Pediatr. 2014; 40: 91 .

[10] Al Darzi W, Al Mudares F, Farah A, Ali A, Marzouk D. Knowledge of periconceptional folic acid use among pregnant women at Ain Shams University Hospital, Cairo, Egypt. East Mediterr Heal J. 2014; 20 (09): 561-8.

[11] Lim E, Lee S, Arzuar N, Rahim A, Azdiana S, Din T. www.eduimed.com Penerbit Universiti Sains Malaysia. Educ Med J [Internet]. 2016; 8 (4): 47-56. Available from: http://dx.doi.org/10.5959/eimj.v8i4.473.

[12] Lawal TA, Adeleye AO. Determinants of folic acid intake during preconception and in early pregnancy by mothers in Ibadan, Nigeria. 2014. Pan Afr Med J: 19: 1-6.

[13] Health Council of the Netherlands: Preconception care: A good beginning. Health Council of the Netherlands. 2007.

[14] United Nations. Millenium develoment goals. 2015; 1-75. Available from: http://www.un.org/millenniumgoals/2015 MDG_Report/pdf/ MDG2015rev(July 1).pdf.

[15] World health organization. maternal-mortalityinfographic2015_part4 who.pdf. geneve, swizerland; 2015.

[16] Mgawadere F, Kana T, Van Den Broek N. Measuring maternal mortality: A systematic review of methods used to obtain estimates of the Maternal Mortality Ratio (MMR) in low- and middle-income countries. Br Med Bull. 2017; 121 (1): $121-34$.

[17] Central Statistical Agency (CSA). Ethiopia Demographic and Health Survey 2016. Addis Ababa, Ethiopia, and Rockville, Maryland, USA: CSA and ICF. P127, 280. 2016.

[18] AtrashHK, Johnson K, AdamsM et al. Preconception care for improving perinatal outcomes: the time to act. Matern Child Health J. 2006; 10: S3-S11. 2006.

[19] Group WB. Trends in Maternal Mortality: 1990 to 2015. 2015.

[20] HSTP F. Health Sector Transformation Plan. 2015.

[21] Organization. WH. Meeting to develop a global consensus on preconception care to reducematernal and childhoodmortality and morbidity. Geneva: World Health Organization; 2013. 2013.

[22] Patabendige M, Goonewardene IMR. Preconception care received by women attending antenatal clinics at a Teaching Hospital in Southern Sri Lanka. Sri Lanka J Obstet Gynaecol. 2013; 35 (1): 3 .

[23] Akinajo OR, Osanyin GE, Okojie OE. DOI: 10.4103/jcls.jcls_3_17.2019; (4): 4-11. 
[24] K. Ahmed, Isra Mutasim Hamad Elbashir, S. Ibrahim, Mohamed, Amel Kamil M. Saeed, A. Alawad. Knowledge, attitude and practice of preconception care among Sudanese women in reproductive age about rheumatic heart disease at Alshaab and Ahmad Gassim hospitals 2014-2015 in Sudan. 2015. Basic Res J Med Clin Sci; 4 (7): 5.

[25] Mutale P, Kwangu M, Chishala MKM, Muchemwa C, Silitongo M, Chileshe M. Knowledge and Preconception Care Seeking Practices among Reproductive-age Diabetic Women in Zambia. Int J Transl Med Res Public Heal. 2017; 1 (2): 3643.

[26] Conlin ML MA, Broadbent JL, Conlin ML, MacLennan AH BJ. Inadequate compliance with periconceptional folic acid supplementation in South Australia. 2006. Aust N Z J Obstet Gynaecol; 46 (6): 528-33.

[27] Lawal TA AA. Determinants of folic acid intake during preconception and in early pregnancy by mothers in Ibadan, Nigeria. 2014. The Pan African Medical Journal; 19.

[28] Sh S, Aghodak P IM. Effect of preconception care protocol implementation on women's awareness. Iranian Journal of Education in Medical science. 2007: 525-32.

[29] Ayalew Y, Mulat A, Dile M, Simegn A. Women's knowledge and associated factors in preconception care in adet, west gojjam, northwest Ethiopia: A community based cross sectional study. Reprod Health [Internet]. 2017; 14 (1): 1-10. Available from: http://dx.doi.org/10.1186/s12978-017-0279-4.

[30] Kassa A, Yohannes Z. Women's knowledge and associated factors on preconception care at Public Health Institution in Hawassa City, South Ethiopia. BMC Res Notes [Internet]. 2018; 11 (1): 1-6. Available from: https://doi.org/10.1186/s13104-018-3951-z.

[31] Asresu TT, Hailu D, Girmay B, Abrha MW. Mothers ' utilization and associated factors in preconception care in northern Ethiopia: a community based cross sectional study. 2019; 1: 1-7.

[32] Demisse TL, Aliyu SA, Kitila SB, Tafesse TT, Gelaw KA, Zerihun MS. Utilization of preconception care and associated factors among reproductive age group women in Debre Birhan town, North Shewa, Ethiopia. Reprod Health. 2019; 16 (1): 1-10.

[33] AT. Knowledge, practice and associated factors of preconception care service among pregnant women attending antenatal care in governmental health facilities, Debremarkos town, Northwest Ethiopia: unpublished thesis. 2015. 2015.

[34] Gezahegn, Andualem. Assessment Knowledge and Experience of Preconception Care Among Pregnant Mothers Attending Antenatal Care in West Shoa Zone Public Health Centers. Biomed Cent Public Heal. 2016; 10 (4): 247-53.
[35] Chepngetich J, Keraka M, Njagi J. Assessment of the Knowledge on Pre Conception Care Among Women of Reproductive Age in Ruiru Sub-County, Kiambu County, Kenya. 2018. Glob J Health Sci.; 3 (1): 82-100.

[36] (CEP) C for effective practice. Preconception Health Care Tool. 2018; (October): 1-5.

[37] Joyce C. Utilization of Preconception Care Services Among Women of. 2018.

[38] Ya G, Tm L, As A. Preconception Care Utilization and its Associated Factors among Pregnant Women in Adet, NorthWestern Ethiopia ( Implication of Reproductive Health) Journal of Women 's Health Care. 2018; 7 (5).

[39] Feleke Daniel. Knowledge, uptake of preconception care and associated factors among reproductive age group women in west shoa zone, Ethiopia. 2018; 1-22.

[40] Nepali G, Sapkota SD. Knowledge and practice regarding preconception care among antenatal mothers. Int J Perceptions Public Heal. 2017; 1 (4): 224-7.

[41] Mirkhan Ahmed H, Jamil Piro T. Knowledge, Practice, and Attitude of Married Women About Preconception Care. J Client-centered Nurs Care. 2017; 3 (1): 37-44.

[42] Jafari F RS. Iranian women's knowledge and attitude regarding preconception health: 12 years after integration into the primary health care network. 2017. J Nurs Midwifery Sci.; 4: 104-9. 2017.

[43] Diabetes and Pregnancy Group F. Knowledge about preconception care in French women with type 1 diabetes. 2005; Vol 31, $\mathrm{N}^{\circ}$.

[44] E, L. C. de J-P, K. M. van der PB, S. le C, W. J. J. A SEB. The Effect of During, Preconception Counseling on Lifestyle and Other Behavior Before and 2008; Pregnancy. 2008. Women's Heal Issues.

[45] Gautam P, Dhakal R. Knowledge on Preconception Care among Reproductive age Women. Saudi J Med Pharm Sci [Internet]. 2016; 2 (1): 1-6. Available from: http://scholarsmepub.com/sjmps/\%5Cnhttp://scholarsmepub.c om/.

[46] Yan D, Xiao-Tian L, Fei X Y-LY. Survey on the implementation of preconception Care in Shanghai, China. Paediatr Perinat Epidemiol. 2015; 29: 492-500.

[47] Al-Akour NA, Sou'Ub R, Mohammad K, Zayed F. Awareness of preconception care among women and men: A study from Jordan. 2015. J Obstet Gynaecol (Lahore); 35 (3): 246-50. 